\title{
The role of stress hormones in the relationship between resting blood pressure and coagulation activity
}

\author{
Petra H. Wirtz ${ }^{a}$, Ulrike Ehlert ${ }^{a}$, Luljeta Emini ${ }^{a}$, Katharina Rüdisüli ${ }^{a}$, \\ Sara Groessbauer ${ }^{\mathrm{a}}$, Brent T. Mausbach ${ }^{\mathrm{c}}$ and Roland von Känel ${ }^{\mathrm{b}}$
}

Background Systemic hypertension confers a

hypercoagulable state. We hypothesized that resting mean blood pressure (MBP) interacts with stress hormones in predicting coagulation activity at rest and with acute mental stress.

Methods We measured plasma clotting factor VII activity (FVII:C), FVIII:C, fibrinogen, D-dimer, epinephrine and norepinephrine, and saliva cortisol in $\mathbf{4 2}$ otherwise healthy normotensive and hypertensive medication-free men (mean age $43 \pm 14$ years) at rest, immediately after stress, and twice during $60 \mathrm{~min}$ of recovery from stress.

Results At rest, the MBP-by-epinephrine interaction predicted FVII:C $(\beta=-0.33, P<0.04)$ and D-dimer $(\beta=0.26, P<0.05)$, and the MBP-by-cortisol interaction predicted $D$-dimer $(\beta=0.43, P=0.001)$, all independent of age and body mass index (BMI). Resting norepinephrine predicted fibrinogen $(\beta=0.42, P<0.01)$ and $D$-dimer $(\beta=0.37, P<0.03)$, both independent of MBP. MBP predicted FVIII:C change from rest to immediately poststress independent of epinephrine $(\beta=-0.37, P<0.03)$ and norepinephrine $(\beta=-0.38, P<0.02)$. Cortisol change predicted FVIII:C change $(\beta=-0.30, P<0.05)$ independent of age, BMI and MBP. Integrated norepinephrine change from rest to recovery (area under the curve, AUC) predicted $D$-dimer AUC $(\beta=0.34, P=0.04)$ independent of MBP. The MBP-by-epinephrine AUC interaction predicted FVII:C AUC
( $\beta=0.28)$ and fibrinogen AUC $(\beta=-0.30)$, and the MBP-bynorepinephrine AUC interaction predicted FVIII:C AUC $(\beta=-0.28)$, all with borderline significance $\left(P_{s}<0.09\right)$ and independent of age and BMI.

Conclusions MBP significantly altered the association between stress hormones and coagulation activity at rest and, with borderline significance, across the entire stress and recovery interval. Independent of MBP, catecholamines were associated with procoagulant effects and cortisol reactivity dampened the acute procoagulant stress response.

Keywords: blood coagulation, catecholamines, cortisol, hypertension, psychological stress

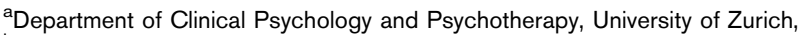
${ }^{b}$ Department of General Internal Medicine, University Hospital Berne, Switzerland and 'Department of Psychiatry, University of California San Diego, USA

Correspondence and requests for reprints to Roland von Känel MD, Professor of Medicine, Department of General Internal Medicine, Division of Psychosomatic Medicine, University Hospital, Inselspital, CH-3010 Bern, Switzerland Tel: +411632 20 19; fax: +413138211 84; e-mail: roland.vonkaenel@insel.ch

Sponsorship: This work was supported by research grant 2003 from the University of Zurich (to P.H.W.).

\section{Introduction}

Virchow's triad of thrombogenesis posits that changes in the vessel wall (e.g. endothelial dysfunction with atherosclerosis), blood flow (e.g. stasis), and blood constituents (e.g. coagulation activation) together contribute to arterial thrombosis [1]. Hypertensive individuals showed elevated levels of coagulation factors and von Willebrand factor (VWF) in plasma, as well as hyperactive platelets on the one hand, and impaired fibrinolysis on the other, compatible with a hypercoagulable state [2]. A number of hemostasis molecules, also increased in hypertension, are now viewed as 'new' risk factors of coronary artery and cerebrovascular disease $[3,4]$. In particular, meta-analyses demonstrate that fibrinogen [5], VWF [6] and D-dimer [7], the latter indicating activation of the entire coagulation system, independently predict coronary artery disease. These data support the notion that the hypercoagulable state in hypertension is of clinical relevance because it could contribute to the increased risk of atherothrombotic events (e.g. myocardial infarction and stroke) in hypertensive individuals [8].

The pathophysiological underpinnings of the hypercoagulable state in hypertension are not fully understood, though target organ damage and endothelial dysfunction might be involved. Fibrinogen and VWF levels relate to left ventricular hypertrophy [9] and nephropathy [10], respectively. Moreover, elevated blood pressure (BP) is a strong risk factor for atherosclerosis [11] which is characterized by an early decline in endothelial function [12]. In hypertension, increased plasma levels of endothelium-derived VWF indicate endothelial damage [13]. The endothelium is crucial in regulating blood flow by providing an active antithrombotic surface and by secreting antithrombotic 
factors into the bloodstream [14]. If perturbed, the endothelium loses its ability to neutralize procoagulant processes, giving rise to a shift in the hemostatic balance between prothrombotic and antithrombotic forces towards hypercoagulability [14].

We aimed to investigate whether hyperactivity of the sympathetic nervous system (SNS) and of the hypothalamic-pituitary-adrenal (HPA) axis would also contribute to procoagulant activity with elevated BP. The reasoning behind this investigation is twofold. First, sympathetic activation by acute mental stress or as mimicked by catecholamine infusions, elicits hypercoagulability [15], and increased HPA axis activity [16] and exogenously administered glucocorticoids [17] also provoked hypercoagulability. Second, the SNS as evidenced by increased muscle sympathetic nerve activity or catecholamine turnover is hyperactive in hypertensives as opposed to normotensives [18]. This difference is not restricted to resting (i.e. steady state) SNS activity but is also observed in the response to sympathetic challenge [19]. For instance, hypertensives showed greater platelet activity [20] and reduced stress-induced fibrinolysis activation [21] in comparison to normotensives. Subtle hyperactivity of the HPA axis has also been postulated in hypertension [22]. However, whether catecholamine or cortisol reactivity contributes to prothrombotic changes in hypertensives has not been investigated.

Elevated BP predicts atherosclerotic risk along a continuum of severity [11] and systolic BP and diastolic BP are both predictive of cardiovascular morbidity [23]. In order to alleviate the risk of random associations with separate analyses for systolic BP and diastolic BP, we therefore investigated the linear relationship between mean blood pressure (MBP) and coagulation measures. We hypothesized that MBP would affect the relationship between resting and stress-induced levels of plasma catecholamines and of salivary free-cortisol and plasma levels of clotting factors VII (FVII:C), VIII (FVIII:C), fibrinogen and $\mathrm{D}$-dimer in a sample of hypertensive and normotensive subjects. We selected these coagulation measures because we recently found them to be responsive to acute psychosocial stress in a group of healthy and normotensive middle-aged men [24] other than those included in the present study. Granted that stress axes are more active with higher BP [18,19,22] and that sympathetic activation kindles procoagulability in a dose-response manner [24-26], we assumed a mechanism by which elevated stress hormones with higher MBP promote greater coagulability at rest, in response to acute stress, and during recovery from stress. The novel aspect of our investigation was therefore to examine in one study whether hormones of both stress axes have a specific effect on different coagulation factors in relation to resting blood pressure level.

\section{Methods}

\section{Study participants}

The Ethics Committee of the State of Zurich, Switzerland, formally approved the research protocol. The final study sample consisted of 48 subjects who provided written informed consent. With the aid of the Swiss Red Cross, Zurich and by advertisement, we recruited non-smoking hypertensive and normotensive men who, other than having hypertension, were in excellent physical and mental health, confirmed by an extensive health questionnaire [16] and telephone interview. Specific exclusion criteria, obtained by subjects' selfreport, were: regular heavy exercise, alcohol and illicit drug abuse; any heart disease, varicosis or thrombotic diseases, elevated blood sugar and diabetes, elevated cholesterol, liver and renal diseases, chronic obstructive pulmonary disease, allergies and atopic diathesis, rheumatic diseases and current infectious diseases. In addition, participants were included only if they reported taking no medication, either regularly or occasionally. If the personal or medication history was not conclusive, the subjects' primary care physician was contacted for clarification.

\section{Assessment of hypertension}

Three seated BP measurements were obtained by sphygmomanometry after a rest of $5 \mathrm{~min}$ on three different days, and the average BP was computed. Following World Health Organization criteria, hypertension was defined as systolic $\mathrm{BP} \geq 140 \mathrm{mmHg}$ and/or diastolic $\mathrm{BP} \geq 90 \mathrm{mmHg}$ [27]. Average MBP was calculated across all individuals using the formula $[2 / 3($ diastolic $\mathrm{BP})+1 / 3$ (systolic BP)].

\section{Stress protocol}

Subjects were tested between 1400 and $1600 \mathrm{~h}$. They had abstained from physical exercise, alcohol and caffeinated beverages since the previous evening. We used the Trier Social Stress Test (TSST) combining a 5-min preparation phase followed by a 5-min mock job-interview, and 5-min mental arithmetic before an audience [28]. The TSST evokes reliable physiological responses in different systems, including coagulation factors investigated in the present study [24]. During recovery, subjects remained seated in a quiet room for $60 \mathrm{~min}$.

Heart rate (HR) was followed continuously via a portable monitor (Polar system, S810; Polar, Finland) and means were computed immediately before stress (i.e. average of $5 \mathrm{~min}$ ), during stress (i.e. average of speech and arithmetic HR) and $20 \mathrm{~min}$ thereafter. Blood pressure was measured immediately before and $20 \mathrm{~min}$ after stress by sphygmomanometry, and continuously during stress (i.e. average of speech and arithmetic BP) by the Vasotrac APM205A device (Medwaves Inc., St. Paul, Minnesota, USA) respectively. Stress measures are presented after 
adjustment to sphygmomanometry readings following methods described previously [24].

Blood for coagulation measures was obtained immediately before stress, immediately after stress, and 20 and $60 \mathrm{~min}$ after stress. At these time points and additionally at $10 \mathrm{~min}$ after stress, blood was drawn to determine catecholamine levels. For cortisol measurements, saliva samples were obtained at rest, immediately after stress and every $10 \mathrm{~min}$ thereafter.

\section{Coagulation assays}

Venous blood was drawn through an indwelling forearm catheter into polypropylene tubes containing 3.8\% sodium citrate. Centrifugation was at $2000 \times \boldsymbol{g}$ for $20 \mathrm{~min}$ at $4^{\circ} \mathrm{C}$. The plasma obtained was immediately aliquoted in polypropylene tubes and frozen at $-80^{\circ} \mathrm{C}$. All analyses of coagulation factors used the BCS Coagulation Analyser (Dade Behring, Liederbach, Germany). Determination of FVII:C and FVIII:C used standard coagulometric methods using factor-deficient standard human plasma and reagents (Dade Behring) and plasma fibrinogen was determined using a modified Clauss method (Multifibren U; Dade Behring). Plasma D-dimer was measured by means of an enzyme-linked immunosorbent assay (Asserachrom Stago, Asnières, France). Inter- and intra-assay coefficients of variation (CVs) were $<10 \%$ for all coagulation measures.

\section{Stress hormone assays}

Venous blood for measuring catecholamines was drawn into EDTA-coated monovettes (Sarstedt, Numbrecht, Germany). Immediately thereafter, samples were centrifuged for $10 \mathrm{~min}$ at $2000 \times \boldsymbol{g}$, with the plasma obtained stored at $-80^{\circ} \mathrm{C}$. Plasma catecholamines were determined by means of high-performance liquid chromatography (HPLC) and electrochemical detection after liquid-liquid extraction [29]. The detection limit was $10 \mathrm{pg} / \mathrm{ml}$ and interand intra-assay $\mathrm{CVs}$ were $<5 \%$ for both catecholamines.

Saliva samples were collected in Salivettes (Sarstedt, Rommelsdorf, Germany) and stored at $-20^{\circ} \mathrm{C}$ until analysis. Centrifugation of thawed samples was at $3000 \times \boldsymbol{g}$ for $10 \mathrm{~min}$, resulting in low-viscosity saliva. Salivary free-cortisol concentrations were measured using a commercial chemiluminescence immunoassay (LIA) with a high sensitivity of $0.16 \mathrm{ng} / \mathrm{ml}$ (IBL Hamburg, Germany) [30]. Inter- and intra-assay $\mathrm{CVs}$ were $<11.5$ and $7.7 \%$, respectively.

\section{Statistical analyses}

Data were analyzed using the SPSS (version 13.0) statistical software package (SPSS Inc., Chicago, Illinois, USA). All tests were two-tailed with level of significance set at $P \leq 0.05$ and level of borderline significance set at $P \leq 0.1$. Prior to statistical analyses all data were tested for normality using the Kolmogorov-Smirnov test. BMI was logarithmically transformed and coagulation values were subject to Blom normal score transformation. For reasons of clarity, we provide untransformed data. We used independent-samples $t$-tests to investigate differences in measures between groups, and the Friedman test to investigate changes in hemodynamic, coagulation and hormone measures across all time points.

Relative change scores in coagulation measures, hormones and hemodynamic factors from rest to immediately after stress were computed by the formula $x=(100 \% / y) \times z$, where $x=$ change score $(\%), y=$ resting value of a variable, and $z=$ stress value of a variable. To compute the cortisol change score, the cortisol level 10 min after stress (i.e. peak response) was used instead of levels immediately after stress. Calculation of the area under the curve (AUC) for coagulation and hormone measures across all time points followed a previous formula that considers the variable time between measurements and ignores the distance from zero for all measurements, thereby emphasizing the integrated changes over time [31]. Change scores and AUC measures for coagulation factors were computed on original data and transformed thereafter.

Hierarchical linear regression analysis was applied using the enter method whereby none of the models considered more than five independent variables, to prevent model overfitting [32]. Following our previous observations [22], the optimal total sample size to detect an expected effect size of 0.35 in regression analyses with a power between 0.80 (maximum of five predictors) and 0.95 (minimum of one predictor) was $n=42$. We regressed MBP, hormone levels and the interaction thereof in this order on coagulation measures. We then tested whether these relationships would be independent of age and BMI, which were entered first into the equation. In order to reduce problems with multicollinearity, all independent variables were centered to the mean, and centered values of MBP and hormone levels were multiplied to obtain interaction terms [33]. A significant interaction would mean that the slope of the relationship between hormones and coagulation factors is different with high $\mathrm{BP}$ (mean centered MBP minus one SD) compared to low BP (mean centered MBP plus one SD). Using the approach described by Holmbeck [34], we performed post-hoc analyses on interaction terms to determine the relationship between hormones and coagulation factors for low and high MBP values. Post-hoc tests were adjusted for age and BMI to identify the extent to which hormones were related to coagulation factors for high and low MBP values [34].

We did not perform statistical adjustment for multiple tests because the various coagulation factors differ substantially in their position and function within the coagulation cascade and, moreover, their dynamics are differently affected by different stress hormones, and 
Table 1 Characteristics of the 42 subjects studied

\begin{tabular}{lccc}
\hline & $\begin{array}{c}\text { Hypertensives } \\
(n=17)\end{array}$ & $\begin{array}{c}\text { Normotensives } \\
(n=25)\end{array}$ & $P$ value \\
\hline $\begin{array}{l}\text { Age (years) } \\
\text { Body mass index } \\
\quad\left(\mathrm{kg} / \mathrm{m}^{2}\right)\end{array}$ & $44.0 \pm 15$ & $43 \pm 14$ & 0.787 \\
$\begin{array}{l}\text { Systolic blood } \\
\text { pressure (mmHg) }\end{array}$ & $27.2 \pm 3.4$ & $25.1 \pm 2.5$ & 0.027 \\
$\begin{array}{l}\text { Diastolic blood } \\
\text { pressure (mmHg) }\end{array}$ & $151 \pm 7$ & $123 \pm 9$ & $<0.001$ \\
$\begin{array}{l}\text { Factor VII clotting } \\
\quad \text { activity (\%) }\end{array}$ & $103 \pm 29$ & $79 \pm 6$ & $<0.001$ \\
$\begin{array}{l}\text { Factor VIII clotting } \\
\quad \text { activity (\%) }\end{array}$ & $106 \pm 26$ & $107 \pm 23$ & 0.617 \\
$\begin{array}{l}\text { Fibrinogen (g/l) } \\
\begin{array}{l}\text { D-dimer (ng/ml) } \\
\text { Norepinephrine } \\
\quad(p g / m l)\end{array}\end{array}$ & $2.8 \pm 0.6$ & $98 \pm 23$ & 0.163 \\
$\begin{array}{l}\text { Epinephrine (pg/ml) } \\
\text { Cortisol (nmol/l) }\end{array}$ & $419 \pm 123$ & $2.5 \pm 0.5$ & 0.044 \\
\hline
\end{tabular}

Values are given as mean \pm SD. Coagulation factor and stress hormone measures reflect plasma levels at rest (i.e. immediately before the stressor).

across the different time intervals assessed [15,24]. In this case of very specific pre-established hypotheses some authors do not advocate statistical adjustment for multiple tests because this may deem truly important associations insignificant [35].

\section{Results}

\section{Subjects' characteristics}

Table 1 provides the characteristics of the 17 hypertensive subjects and 25 normotensive controls studied. As per definition, hypertensive subjects had higher systolic and diastolic BP than normotensive subjects. In addition, hypertensives had higher BMI, and higher plasma levels of fibrinogen and of norepinehrine at rest (i.e. immediately before stress) than normotensives.

\section{Coagulation activity at rest Epinephrine at rest}

FVII: $C$ at rest. There was an interaction between epinephrine and MBP for FVII:C $(\beta=-0.33, P=0.045$; $\left.\Delta R^{2}=0.101\right)$, which was maintained $(\beta=-0.33$, $\left.P=0.037 ; \quad \Delta R^{2}=0.095\right) \quad$ when controlling for age $(P=0.22)$ and BMI $(\beta=-0.35, P=0.037)$. There was no significant correlation between epinephrine and FVII:C with high $\operatorname{MBP}(\beta=-0.01, P=0.96)$ and with low $\operatorname{MBP}(\beta=0.17, P=0.31)$, respectively.

FVIII:C at rest. Epinephrine predicted FVIII:C $(\beta=0.33$, $\left.P=0.046 ; \Delta R^{2}=0.123\right)$ independent of age $(P=0.52)$, BMI $(P=0.42)$, and $\operatorname{MBP}(P=0.13)$.

$D$-dimer at rest. Epinephrine interacted with $\mathrm{MBP}$ in predicting D-dimer $\left(\beta=0.26, P=0.042 ; \Delta R^{2}=0.062\right)$ independent of age $(\beta=0.27, P=0.060)$ and $\mathrm{BMI}$ $(\beta=0.49, \quad P=0.001)$. Epinephrine was unrelated to D-dimer when MBP was low $(\beta=0.09, P=0.54)$ but predicted D-dimer with borderline significance when MBP was high $(\beta=0.23, P=0.067)$.

\section{Norepinephrine at rest}

Fibrinogen at rest. The relationship between norepinephrine and fibrinogen was significant $(\beta=0.42$, $\left.P=0.008 ; \Delta R^{2}=0.149\right)$ independent of $\operatorname{MBP}(P=0.14)$ and became of borderline significance $(\beta=0.28$, $P=0.060)$ when also controlling for age $(P=0.56)$ and BMI $(\beta=0.37, P=0.016)$.

$D$-dimer at rest. Norepinephrine predicted D-dimer $\left(\beta=0.37, P=0.028 ; \Delta R^{2}=0.117\right)$ independent of $\mathrm{MBP}$ $(P=0.67)$; this relationship became non-significant $(P=0.24)$ with additional adjustment for age $(P=0.15)$ and BMI $(\beta=0.46, P=0.003)$.

\section{Cortisol at rest}

FVIII: $C$ at rest. MBP predicted FVIII:C $(\beta=0.32$, $\left.P=0.048 ; \quad R^{2}=0.084\right) \quad$ independent of cortisol $(P=0.66)$ with this relationship becoming of borderline significance $(\beta=0.30, P=0.086)$ when additionally controlling for age $(P=0.76)$ and BMI $(P=0.55)$.

Fibrinogen at rest. MBP predicted fibrinogen $(\beta=0.39$, $\left.P=0.014 ; R^{2}=0.143\right)$ independent of cortisol $(P=0.23)$; this relationship became non-significant $(P=0.12)$ when age $(P=0.31)$ and $\mathrm{BMI}(\beta=0.43, P=0.006)$ were also controlled for.

$D$-dimer at rest. There was an interaction between cortisol and $\mathrm{MBP}$ in predicting D-dimer $(\beta=0.37, P=0.025$; $\left.\Delta R^{2}=0.120\right)$, which was maintained $(\beta=0.43$, $\left.P=0.001 ; \quad \Delta R^{2}=0.160\right)$ when controlling for age $(\beta=0.24, P=0.053)$ and BMI $(\beta=0.54, P<0.001)$. Cortisol did not significantly predict $\mathrm{D}$-dimer with high MBP $(\beta=0.10, P=0.37)$ but showed an inverse relationship with $\mathrm{D}$-dimer of borderline significance with low $\operatorname{MBP}(\beta=-0.24, P=0.091)$.

\section{Hemodynamic, stress hormone and coagulation reactivity}

There were significant time effects for hemodynamic measures $(P<0.001 ;$ Fig. 1a-c), stress hormones $(P<0.001$; Fig. $1 \mathrm{~d}-\mathrm{f})$ and coagulation factors $(P<0.001$; Fig. $2 \mathrm{a}-\mathrm{d}$ ), indicating a significant stress response in all of these variables. Post-hoc analyses revealed that increases in hemodynamic measures, stress hormones and coagulation factors from rest to immediately after stress was significant (all $P<0.001$ ).

\section{Coagulation change from rest to immediately after stress}

\section{Epinephrine change}

FVIII:C change. MBP predicted FVIII:C change $\left(\beta=-0.37, P=0.023 ; R^{2}=0.147\right)$ independent of epinephrine change $(P=0.92)$. This relationship became of borderline significance $(\beta=-0.28, P=0.090)$ with additional controlling for age $(P=0.099)$ and BMI $(P=0.49)$. 
(a) Heart rate change

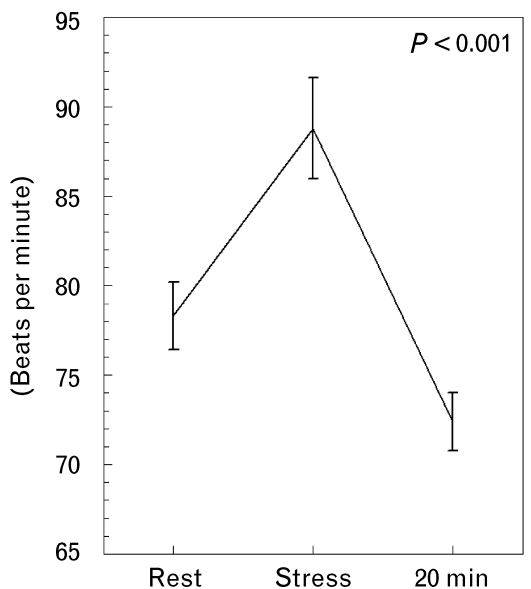

(d)

Norepinephrine change

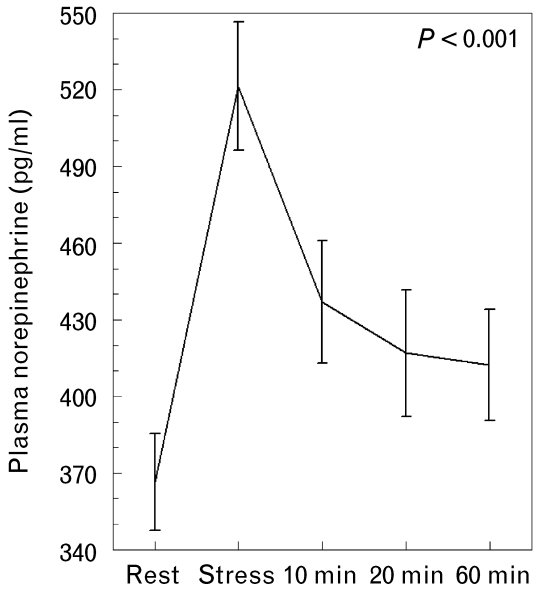

(b) Systolic blood pressure change

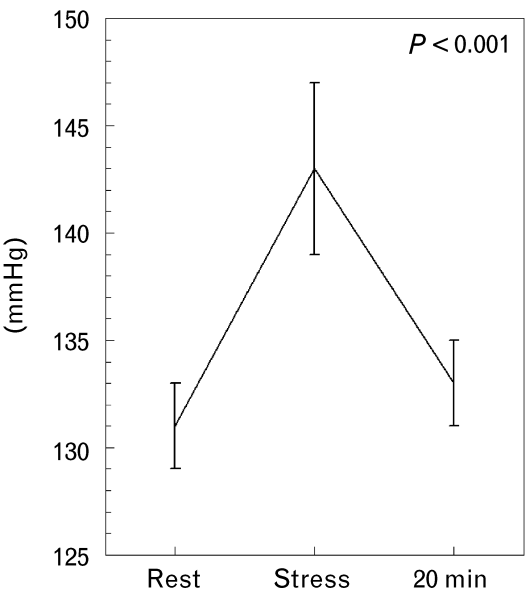

(e)

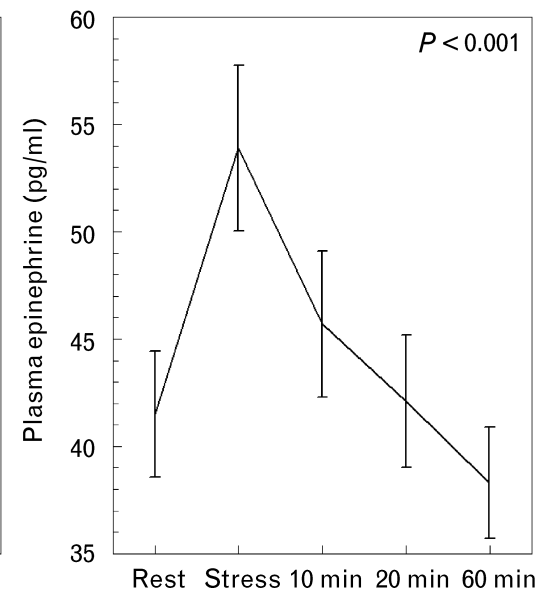

(c)

Diastolic blood pressure change

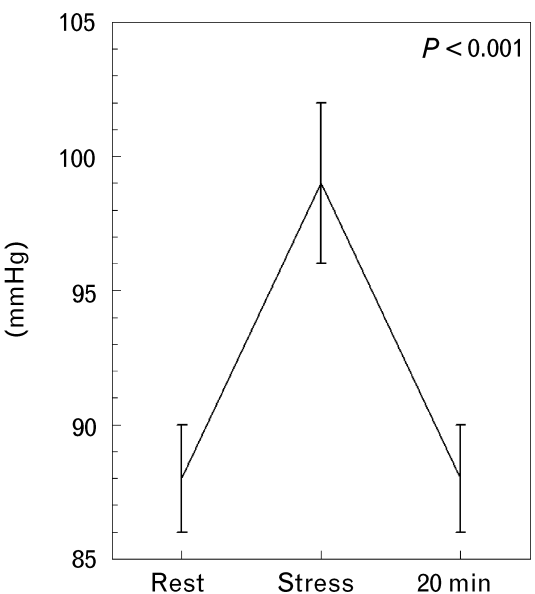

(f)

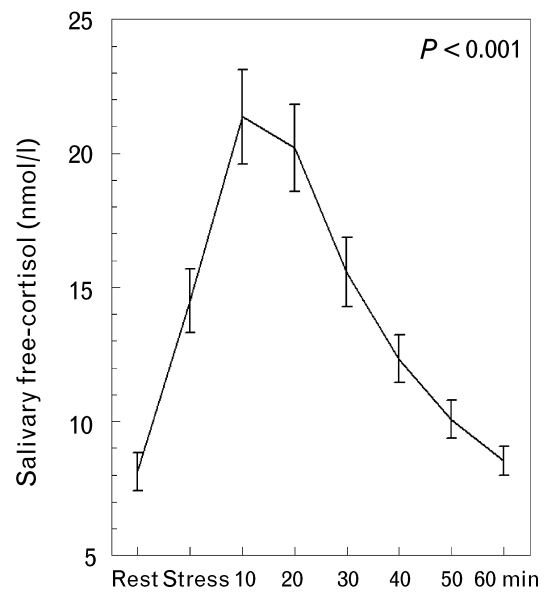

Hemodynamic and stress hormone levels over time. Values are means \pm SEM. Across all 42 subjects, the stressor elicited a significant response in hemodynamic measures $(a-c)$ and hormone levels $(d-f)$.

\section{Norepinephrine change}

FVIII:C change. MBP predicted FVIII:C change $\left(\beta=-0.38, P=0.015 ; R^{2}=0.147\right)$ independent of norepinephrine change $(P=0.75)$ with this relationship reaching borderline significance $(\beta=-0.28, P=0.073)$ when age $(P=0.16)$ and BMI $(P=0.31)$ were additionally controlled for.

D-dimer change. Norepinephrine change predicted Ddimer change independent of MBP $\quad(\beta=0.32$, $\left.P=0.049 ; \quad \Delta R^{2}=0.101\right)$. However, this relationship became non-significant $(P=0.12)$ when controlling for age $(P=0.22)$ and BMI $(P=0.15)$.

\section{Cortisol change}

FVIII: $C$ change. $\mathrm{MBP}\left(\beta=-0.31, P=0.042 ; R^{2}=0.146\right)$ and cortisol change $\left(\beta=-0.34, P=0.027 ; \Delta R^{2}=0.103\right)$ significantly predicted FVIII:C independent of each other. After adjustment for age $(P=0.091)$ and BMI $(P=0.81), \quad$ cortisol change $(\beta=-0.30, \quad P=0.049$, $\left.\Delta R^{2}=0.078\right)$, but not $\mathrm{MBP}(P=0.095)$, was maintained as a significant predictor of FVIII:C change.

\section{Coagulation change between rest and $60 \mathrm{~min}$ after stress}

Epinephrine area under the curve

FVII:C AUC. There was an interaction between epinephrine AUC and MBP for FVII:C AUC of borderline significance $(\beta=0.30, P=0.069)$ which was maintained $(\beta=0.28, P=0.10)$ when controlling for age $(P=0.50)$ and $\mathrm{BMI}(P=0.30)$. There was no significant relationship between epinephrine AUC and FVII:C AUC with high $(\beta=0.23, \quad P=0.25)$ and with low $(\beta=0.01$, $P=0.95) \mathrm{MBP}$. 
(a) Factor VII clotting activity change

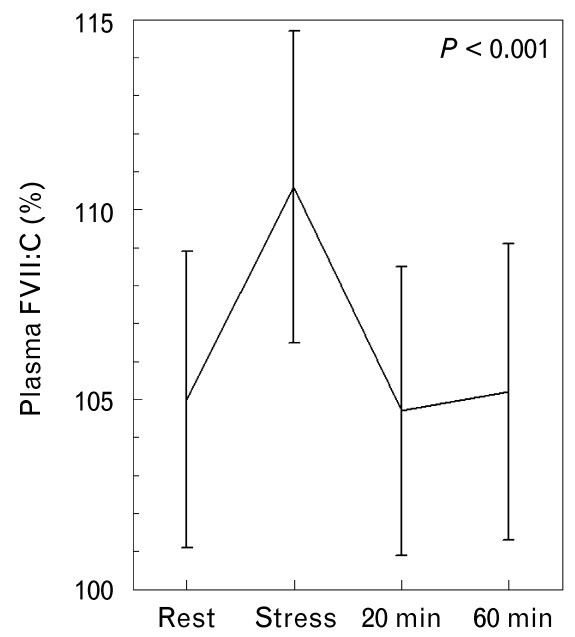

(c)

Fibrinogen change

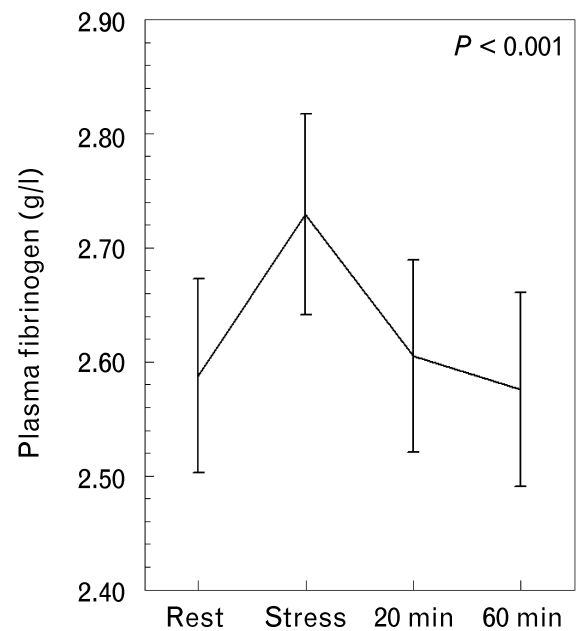

(b)

Factor VIII clotting activity change

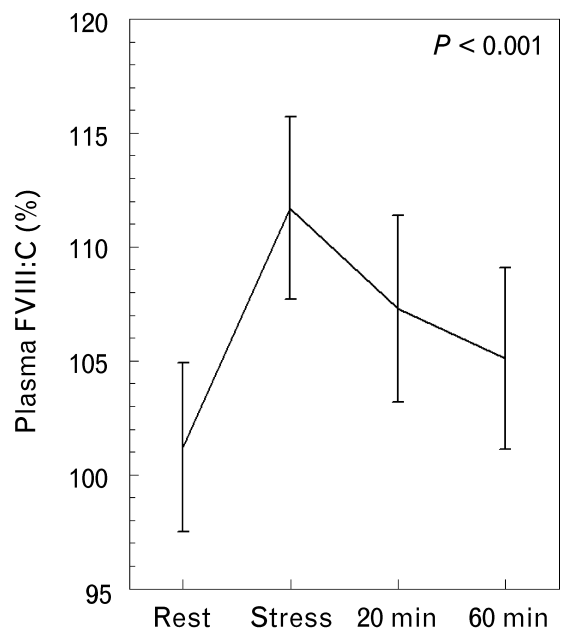

(d)

D-dimer change

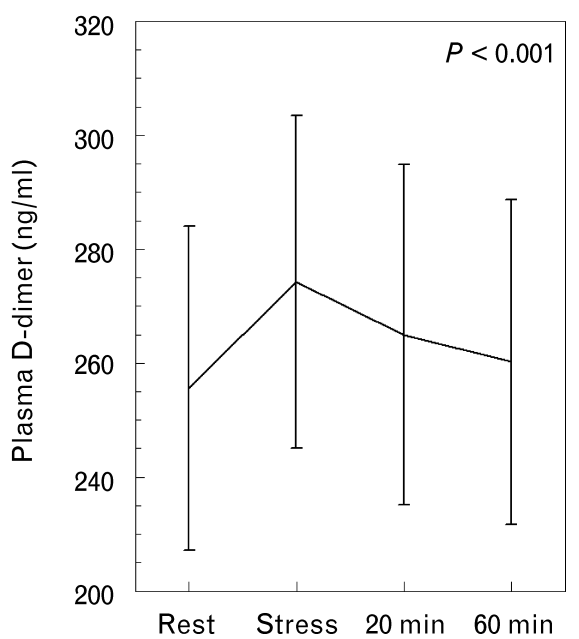

Coagulation factor levels over time. Values are given as means \pm SEM. Across all 42 subjects, the stressor elicited a significant response in plasma levels of FVII:C (a), FVIII:C (b), fibrinogen (c) and D-dimer (d).

Fibrinogen AUC. Epinephrine AUC interacted with MBP in predicting fibrinogen AUC with borderline significance $(\beta=-0.30, P=0.076)$ when controlling for age $(P=0.13)$ and BMI $(P=0.60)$. There was no significant relationship between epinephrine AUC and fibrinogen AUC with high $(\beta=-0.04, P=0.85)$ and with low $(\beta=0.20, P=0.31) \mathrm{MBP}$.

\section{Norepinephrine area under the curve}

FVIII:C AUC. There was an interaction between norepinephrine AUC and MBP for FVIII:C AUC of borderline significance $(\beta=-0.28, P=0.088)$ independent of age $(P=0.76)$ and BMI $(P=0.053)$. Associations between norepinephrine AUC and FVIII:C AUC were not signifi- cant with high $\mathrm{MBP}(\beta=-0.24, P=0.23)$ and with low $\operatorname{MBP}(\beta=-0.07, P=0.71)$.

$D$-dimer AUC. Norepinephrine AUC significantly predicted D-dimer AUC $\left(\beta=0.34, P=0.040, \Delta R^{2}=0.122\right)$ independent of MBP $(P=0.81)$. Additional adjustment for age $(\beta=0.47, P=0.010)$ and BMI $(P=0.096)$ rendered this relationship non-significant $(P=0.57)$.

\section{Discussion}

We found significant interactions between stress hormones and MBP for coagulation measures at rest and with borderline significance, during the entire stress and recovery interval. With high MBP, the slopes of the relationship between resting epinephrine and FVII:C 
and D-dimer, respectively, and between resting cortisol and D-dimer were significantly different from the respective slopes with low MBP. Examination of the beta coefficients suggests that with high MBP, epinephrine approached a statistically significant relationship with D-dimer $(\beta=0.23)$. However, with low MBP, this relationship was much weaker $(\beta=0.09)$. Therefore, it appears that epinephrine poses a greater risk for coagulation activity among those with high BP than among those with low BP. Moreover, post-hoc analyses indicate a positive, albeit non-significant, relationship between cortisol and D-dimer $(\beta=0.10)$ when MBP is high. But, when MBP is low, the correlation is negative $(\beta=-0.24)$, suggesting that cortisol has some mild effect on coagulation activation among high-BP individuals. Together with the associations between resting epinephrine and FVIII:C levels and between resting norepinephrine and fibrinogen levels, our study suggests that stress hormone activity plays a role in regulating steadystate coagulation activity. Importantly, we found that the effect of resting stress hormones on coagulation factors was dependent in part upon MBP level, but was generally not affected by age and BMI.

We tested the effect of stress hormones on stresstriggered coagulation activation in two different ways: in terms of short-term change from rest to immediately after stress, and in terms of overall reactivity including the recovery period. Performing both these analyses exceeds an exploratory investigation because they track quite different aspects in reactivity of the physiological systems under study. There were no interactions between short-term hormone changes and MBP in terms of any coagulation factor. Nonetheless, a greater amount of acute norepinephrine release predicted a higher level of acute D-dimer formation. This notion is consistent with early studies showing that catecholamine infusion dosedependently elicited hemostatic changes in plasma within minutes $[25,26]$ consequent to a rapid release of hemostatic molecules from extravascular compartments into the circulation through a $\beta_{2}$-adrenergic mechanism [15]. Given previous research on greater stress procoagulant reactivity in hypertensives than in normotensives $[20,21]$, the negative association between MBP and the increase of FVIII:C from rest to immediately after stress independent of epinephrine and norepinephrine change was rather unexpected. Part of this observation might relate to the confounding effect of age and BMI, being supported by the finding that the expected direct relationship between norepinephrine and D-dimer change became non-significant with adjustment for age and BMI. Counterintuitively, cortisol and FVIII:C change showed an inverse relationship, suggesting that HPA axis activity was associated with a relatively attenuated shortterm FVIII:C response to stress. One explanation of this finding could relate to research showing that endogenous glucocorticoids may suppress SNS activity [36]. Interest- ingly, in all other analyses, we observed no relationship between cortisol and coagulation activity, either at rest or in relation to acute stress, suggesting that, of the stressmediating systems, the SNS may be of greater importance than the HPA axis in moderating coagulation.

Although not investigated in the present study, sympathetic activation increases platelet activity [20] and decreases fibrinolysis [21] more in hypertensives than in normotensives. It is not known, however, whether such a group difference in thrombogenicity would carry over to recovery from stress. This seems of clinical importance because sustained hypercoagulability during a relatively long period of recovery from stress may be of greater clinical importance than more short-term procoagulant changes rapidly returning to resting levels after stress termination [24]. In contrast to the aforementioned lack of a role of MBP in the relationship between acute changes in stress hormones and coagulation factors, we found that MBP interacted with the integrated release of epinephrine between rest and $1 \mathrm{~h}$ after stress in predicting integrated change of FVII:C and of fibrinogen. In addition, MBP interacted with integrated norepinephrine release in predicting integrated FVIII:C change. We note that, although independent of age and BMI, these relationships reached only borderline significance, and must therefore be regarded as preliminary, pending further replication in larger samples. Again corroborating a role for SNS activity in hypercoagulability, norepinephrine spillover between rest and $1 \mathrm{~h}$ after stress was directly associated with D-dimer formation during this time interval, with adjustment for covariates rendering this relationship non-significant.

The recruitment of apparently healthy and drug-free subjects with reasonable health habits was a strength of our study, because coagulation is affected by numerous diseases, medication (including antihypertensives) and lifestyle factors [15]. Negligible confounding of our coagulation results was further achieved by considering age and BMI a priori as control variables. We mention four limitations of our study. First, even though catecholamine infusion consistently elicited coagulation activation in previous studies [25,26], the significant associations between stress hormones and coagulation factors, as related to MBP, do not prove a causal link in our study. Second, to prevent spurious associations by chance, some investigators would require higher levels of significance than we observed in terms of some associations between stress hormones and coagulation measures. However, it seems acceptable not to adjust for multiple comparisons, given our pre-specified hypotheses [35]. Third, conclusions in terms of whether one stress hormone would affect a particular coagulation factor more specifically than any other must be drawn with caution, given the rather small sample size. Fourth, our findings cannot be generalized to populations other 
than apparently healthy men with BP in the normotensive and mildly hypertensive range. The role of the SNS for high $\mathrm{BP}$ seems most evident in borderline hypertensives [37], whereas hypercoagulability appears to increase with increasing severity of hypertension and end organ damage $[9,10]$, respectively. We were unable to scrutinize a possibly different role of stress hormones in relation to the hypercoagulable state between groups having different severity of hypertension or in women.

To sum up, we found some evidence for our hypothesis that stress hormones affect coagulation activity in relation to screening blood pressure level. This observation appeared to be stronger at rest than in response to acute psychosocial stress. The effect of immediate stress hormone reactivity on coagulation factors was unrelated to BP. Moreover, it is of potential clinical interest that reactivity of hormones predicted coagulation changes during stress recovery depending on screening BP. However, this finding requires replication in larger samples.

\section{References}

1 Lip GY, Blann AD. Does hypertension confer a prothrombotic state? Virchow's triad revisited. Circulation 2000; 101:218-220.

2 Varughese GI, Lip GY. Is hypertension a prothrombotic state? Curr Hypertens Rep 2005; 7:168-173.

3 Folsom AR. Hemostatic risk factors for atherothrombotic disease: an epidemiologic view. Thromb Haemost 2001; 86:366-373.

4 Catto AJ, Grant PJ. Risk factors for cerebrovascular disease and the role of coagulation and fibrinolysis. Blood Coagul Fibrinolysis 1995; 6:497-510.

5 Maresca G, Di Blasio A, Marchioli R, Di Minno G. Measuring plasma fibrinogen to predict stroke and myocardial infarction: an update. Arterioscler Thromb Vasc Biol 1999; 19:1368-1377.

6 Danesh J, Wheeler JG, Hirschfield GM, Eda S, Eiriksdottir G, Rumley A, et al. $\mathrm{C}$-reactive protein and other circulating markers of inflammation in the prediction of coronary heart disease. N Engl J Med 2004; 350:1387-1397.

7 Danesh J, Whincup P, Walker M, Lennon L, Thomson A, Appleby P, et al. Fibrin D-dimer and coronary heart disease: prospective study and metaanalysis. Circulation 2001; 103:2323-2327.

8 Lip GY. Hypertension and the prothrombotic state. J Hum Hypertens 2000; 14:687-690.

9 Lip GY, Blann AD, Jones AF, Lip PL, Beevers DG. Relation of endothelium, thrombogenesis, and hemorheology in systemic hypertension to ethnicity and left ventricular hypertrophy. Am J Cardiol 1997; 80:1566-1571.

10 Pedrinelli R, Giampietro O, Carmassi F, Melillo E, Dell'Omo G, Catapano G, et al. Microalbuminuria and endothelial dysfunction in essential hypertension. Lancet 1994; 344:14-18.

11 MacMahon S, Peto R, Cutler J, Collins R, Sorlie P, Neaton J, et al. Blood pressure, stroke, and coronary heart disease. Part 1, Prolonged differences in blood pressure: prospective observational studies corrected for the regression dilution bias. Lancet 1990; 335:765-774.

12 McGorisk GM, Treasure CB. Endothelial dysfunction in coronary heart disease. Curr Opin Cardiol 1996; 11:341-350.

13 Felmeden DC, Spencer CG, Chung NA, Belgore FM, Blann AD, Beevers DG, Lip GY. Relation of thrombogenesis in systemic hypertension to angiogenesis and endothelial damage/dysfunction (a substudy of the Anglo-Scandinavian Cardiac Outcomes Trial [ASCOT]). Am J Cardiol 2003; 92:400-405.

14 Cines DB, Pollak ES, Buck CA, Loscalzo J, Zimmerman GA, McEver RP, et al. Endothelial cells in physiology and in the pathophysiology of vascular disorders. Blood 1998; 91:3527-3561.

15 Preckel D, von Känel R. Regulation of hemostasis by the sympathetic nervous system: any contribution to coronary artery disease? Heart Drug $2004 ; 4: 123-130$

16 von Känel R, Kudielka BM, Abd-el-Razik A, Gander ML, Frey K, Fischer JE. Relationship between overnight neuroendocrine activity and morning haemostasis in working men. Clin Sci 2004; 107:89-95.

17 Brotman DJ, Girod JP, Posch A, Jani JT, Patel JV, Gupta M, et al. Effects of short-term glucocorticoids on hemostatic factors in healthy volunteers. Thromb Res 2006; 118:247-252.
18 Mancia G, Grassi G, Giannattasio C, Seravalle G. Sympathetic activation in the pathogenesis of hypertension and progression of organ damage. Hypertension 1999; 34:724-728.

19 Fossum E, Hoieggen A, Reims HM, Moan A, Rostrup M, Eide I, Kjeldsen SE. High screening blood pressure is related to sympathetic nervous system activity and insulin resistance in healthy young men. Blood Press 2004; 13:89-94.

20 Tomoda F, Takata M, Kagitani S, Kinuno H, Yasumoto K, Tomita S, Inoue H. Different platelet aggregability during mental stress in two stages of essential hypertension. Am J Hypertens 1999; 12:1063-1070.

21 Palermo A, Bertalero P, Pizza N, Amelotti R, Libretti A. Decreased fibrinolytic response to adrenergic stimulation in hypertensive patients. J Hypertens Supp/ 1989; 7:S162-S163.

22 Whitworth JA, Mangos GJ, Kelly JJ. Cushing, cortisol, and cardiovascular disease. Hypertension 2000; 36:912-916.

23 Safar ME, London GM. Therapeutic studies and arterial stiffness in hypertension: recommendations of the European Society of Hypertension The Clinical Committee of Arterial Structure and Function Working Group on Vascular Structure and Function of the European Society of Hypertension. J Hypertens 2000; 18:1527-1535.

24 von Känel R, Preckel D, Zgraggen L, Mischler K, Kudielka BM, Haeberli A Fischer JE. The effect of natural habituation on coagulation responses to acute mental stress and recovery in men. Thromb Haemost 2004; 92:1327-1335.

25 von Känel R, Dimsdale JE. Effects of sympathetic activation by adrenergic infusions on hemostasis in vivo. Eur J Haematol 2000; 65:357-369.

26 von Känel R, Dimsdale JE, Adler KA, Dillon E, Perez CJ, Mills PJ. Effects of nonspecific beta-adrenergic stimulation and blockade on blood coagulation in hypertension. J App/ Physiol 2003; 94:1455-1459.

27 Kjeldsen SE, Erdine S, Farsang C, Sleight P, Mancia G. 1999 WHO/ISH Hypertension Guidelines subcommittee 1999 WHO/ISH Hypertension Guidelines - highlights and ESH update. J Hypertens 2002; 20:153-155.

28 Kirschbaum C, Pirke KM, Hellhammer DH. The 'Trier Social Stress Test' a tool for investigating psychobiological stress responses in a laboratory setting. Neuropsychobiology 1993; 28:76-81.

29 Ehrenreich H, Schuck J, Stender N, Pilz J, Gefeller O, Schilling L, et al. Endocrine and hemodynamic effects of stress versus systemic CRF in alcoholics during early and medium term abstinence. Alcohol Clin Exp Res 1997; 21:1285-1293.

30 Westermann J, Demir A, Herbst V. Determination of cortisol in saliva and serum by a luminescence-enhanced enzyme immunoassay. Clin Lab 2004; 50:11-24.

31 Pruessner JC, Kirschbaum C, Meinlschmid G, Hellhammer DH. Two formulas for computation of the area under the curve represent measures of total hormone concentration versus time-dependent change. Psychoneuroendocrinology 2003; 28:916-931.

32 Babyak MA. What you see may not be what you get: a brief, nontechnical introduction to overfitting in regression-type models. Psychosom Med 2004; 66:411-421.

33 Kraemer HC, Wilson GT, Fairburn CG, Agras WS. Mediators and moderators of treatment effects in randomized clinical trials. Arch Gen Psychiatry 2002; 59:877-883.

34 Holmbeck GN. Post-hoc probing of significant moderational and mediational effects in studies of pediatric populations. $J$ Pediatr Psycho 2002; 27:87-96

35 Perneger TV. What's wrong with Bonferroni adjustments. BMJ 1998; 316:1236-1238.

36 Kvetnansky R, Fukuhara K, Pacak K, Cizza G, Goldstein DS, Kopin IJ. Endogenous glucocorticoids restrain catecholamine synthesis and release at rest and during immobilization stress in rats. Endocrinology 1993; 133:1411-1419.

37 Julius S, Johnson EH. Stress, autonomic hyperactivity and essential hypertension: an enigma. J Hypertens Supp/ 1985; 3:S11-S17. 